INPLASY

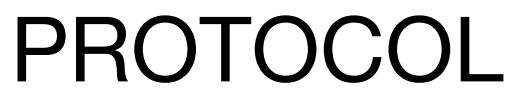

To cite: Zhao et al. Traditional Chinese Medicine for Helicobacter pylori infection: A protocol for a systematic review and meta-analysis. Inplasy protocol 2020120057. doi:

10.37766/inplasy2020.12.0057

Received: 10 December 2020

Published: 10 December 2020

Corresponding author: Jiang Yong

jiangyong@pyztcm.com

Author Affiliation: Chengdu University of Traditional Chinese Medicine, School of Basic Medical Sciences

Support: Grant No: 61801058.

Review Stage at time of this submission: Preliminary searches.

Conflicts of interest:

None.

\section{Traditional Chinese Medicine for Helicobacter pylori infection: A protocol for a systematic review and meta-analysis}

Zhao, Mㅍ Jiang, YC2; Chen, ZX33; Fan, ZP4; Jiang, Y5.

Review question / Objective: This review aims to systematically evaluate the efficacy and safety of traditional Chinese medicine for Helicobacter pylori infection reported in randomized clinical trials (RCTs).

Condition being studied: Helicobacter pylori infection: digestive system, pathogens, stomach environment. Information sources: Web of Science, PubMed, Science Direct, Wan Fang Data Knowledge Service Platform, Chinese Biomedical Literature Database (CBM), Chinese Scientific Journal Database (VIP database), China National Knowledge Infrastructure (CNKI) and EMBASE, which should be published from inception to December 2020.

INPLASY registration number: This protocol was registered with the International Platform of Registered Systematic Review and Meta-Analysis Protocols (INPLASY) on 10 December 2020 and was last updated on 10 December 2020 (registration number INPLASY2020120057).

\section{INTRODUCTION}

Review question / Objective: This review aims to systematically evaluate the efficacy and safety of traditional Chinese medicine for Helicobacter pylori infection reported in randomized clinical trials (RCTs).
Condition being studied: Helicobacter pylori infection: digestive system, pathogens, stomach environment.

\section{METHODS}

Participant or population: All patients who have been diagnosed with Helicobacter 
pylori will be included. There are no restrictions on age, sex, region, race, belief, race, origin and disease course.

Intervention: The experimental group mainly treated Helicobacter pylori infection with traditional Chinese medicine. The use of traditional Chinese medicine is limited to prescription medicines and proprietary Chinese medicines. Prescription drugs need a definite dose, but there are no restrictions on the ingredients, dosage form and doses. For the dosage, such as decoction, granule, pill, powder, etc., other types of traditional Chinese medicine treatment, such as traditional Chinese medicine injections, acupuncture, massage, cupping, etc., will not be included.

Comparator: The control group was given conventional western medicines combined with antibiotics, proton pump inhibitors and aluminate preparations. There are no restrictions on specific drugs, doses and methods. If the control group is treated with Chinese medicine, the study will be excluded.

Study designs to be included: This study includes all relevant randomized controlled trials (RCTs) of traditional Chinese medicine therapy for GC published in Chinese or English, regardless of allocation hidden or blinded.

Eligibility criteria: Randomized controlled trials (RCTs) will be included in this study. There were no limitations on year of publication, publication status.

Information sources: Web of Science, PubMed, Science Direct, Wan Fang Data Knowledge Service Platform, Chinese Biomedical Literature Database (CBM), Chinese Scientific Journal Database (VIP database), China National Knowledge Infrastructure (CNKI) and EMBASE, which should be published from inception to December 2020.

Main outcome(s): The DOI value of $\mathrm{C} 13$ and C14 breath test decreased or was negative, the antibody of Helicobacter pylori in serum, bacterial culture in gastric mucosa and enzyme test in uremia were negative.

Quality assessment / Risk of bias analysis: In order to assess the methodological quality of the included studies, two reviewers (MZ and ZF) will independently use the Cochrane risk of bias tool to examine 7 aspects: random sequence generation, allocation hiding, blindness of participants and personnel, blindness of result evaluation, incomplete data Evaluations, selective results reports and other sources of bias.

Strategy of data synthesis: Pairwise metaanalyses. Using Revman 5.3 software provided by Cochrane Collaboration Network, meta-analyses were carried out for the included researches.

Subgroup analysis: To identify substantial heterogeneity, subgroup analysis will be implemented according to characteristics of patients, type of intervention and outcome measures.

Sensibility analysis: Assess the sensitivity of results by analyzing studies including patients without complications, the studies without missing data.

\section{Country(ies) involved: China.}

Keywords: Helicobacter pylori, Traditional Chinese medicine, protocol, systematic review and meta-analysis.

Contributions of each author:

Author 1 - Zhao Mao.

Email: 1062844276@qq.com

Author 2 - Jiang Yuchang.

Email: 198543912@qq.com

Author 3 - Chen Zhaoxing.

Email: 1172538390@qq.com

Author 4 - Fan Zhipeng.

Email: 156183951@qq.com

Author 5 - Jiang Yong.

Email: jiangyong@pyztcm.com 